\title{
Construcción de nano-dosímetro para el control automático de la dosificación
}

\author{
Midory Esmeralda Vigueras Velázquez, Romeo Urbieta Parrazales, \\ Karen Alicia Aguilar Cruz, José de Jesús Medel Juárez \\ Instituto Politécnico Nacional, Centro de Investigación en Computación, \\ Ciudad de México, México \\ midory.vigueras@gmail.com, rurbieta700@gmail.com, \\ karen_ali320@hotmail.com, jjmedelj@gmail
}

\begin{abstract}
Resumen. Un aspecto clave en la optimización del diseño de un sistema es la selección de los materiales, sensores y métodos que mejor satisfacen las necesidades de diseño, asegurando el máximo rendimiento y el mínimo costo. El enfoque del control automático para la dosificación de líquidos presentado en el este trabajo, consiste de dos fases de desarrollo: a) el diseño y simulación del sistema de control inteligente $\mathrm{y}, \mathrm{b}$ ) la implementación del prototipo del sistema de control inteligente; este último se llevó a cabo con flujo de líquidos de baja viscosidad contenido desde un recipiente cisterna $(60 \mathrm{ml})$ a otro de menor capacidad $(20 \mathrm{ml})$ usando componentes de micro tecnologías avanzadas como son los Sistemas-Micro-Electro-Mecánicos (MEM). El artículo servirá como una guía de referencia y recurso útil para quienes participan en el diseño y fabricación de microsistemas para la dosificación de líquidos mediante actuadores y dispositivos MEM. Cabe mencionar que el diseño en dosificadores de líquidos de baja viscosidad tiene diversas aplicaciones, tanto en la industria automotriz, en dispositivos de electrónica de consumo, en medicina y tecnologías relacionadas con la salud, etc., donde la calidad, precisión, confiabilidad, tamaño, capacidades micrométricas de la mezcla son de suma importancia para el consumidor.
\end{abstract}

Palabras clave: control inteligente de líquidos, MEM, Arduino, Raspberry Pi 3.

\section{Construction of Nano-dosimeter for the Automatic Control of Dosing}

\begin{abstract}
A key aspect in optimizing the design of a system is the selection of materials, sensors and methods that best meet design needs, ensuring maximum performance and minimum cost. The automatic control approach for the dosing of liquids presented in this paper, consists of two phases of development: a) the design and simulation of the intelligent control system and, b) the implementation of the prototype of the intelligent control system; The latter was carried out with a flow of low viscosity liquids from a cistern $(60 \mathrm{ml})$ to a smaller one $(20 \mathrm{ml})$ using advanced micro-technology components such as Micro-Electro-
\end{abstract}


Mechanical Systems (MEM). The article will serve as a reference guide and useful resource for those involved in the design and manufacture of microsystems for the dosing of liquids by means of actuators and MEM devices. It should be mentioned that the design of low viscosity liquid dispensers has various applications, both in the automotive industry, in consumer electronics devices, in medicine and health-related technologies, etc., where quality, precision, reliability, size, micrometric capabilities of the mix are of utmost importance to the consumer.

Keywords: intelligent liquid control, MEM, Arduino, Raspberry Pi 3.

\section{Introducción}

Hoy en día el controlador de fluidos de baja viscosidad tiene gran influencia y aplicación en diversos campos industriales, sobre todo en la industria militar. La física de fluidos interactúa directa con el aire o con el agua, e indirectamente a través de muchos sistemas fluídicos que ellos incorporan. El objetivo del trabajo es mejorar la eficiencia o bien el desempeño a través de calcular los parámetros del sistema y verificarlos en la simulación, así como embeber el sistema de Control Inteligente en un dispositivo digital, con la intención de controlar el flujo, para atrasar o avanzar la transición, suprimir, mejorar la turbulencia, prevenir, o promover la separación. Como resultado se observa la reducción de resistencia, mejora la elevación, aumento de mezcla, mejora la transferencia de calor y supresión de los ruidos inducidos, etc. [1,2,3].

En la mayoría de los casos de fluidos se trabaja en la capa delgada del fluido, de escasos milímetros de grosor, conocida como capa límite, que se forma entre los componentes del vehículo y el fluido circundante (véase la Fig. 1). La posibilidad de crear tecnología a estas dimensiones ha llevado a los pioneros de MEM a desarrollar componentes que se usan en los sistemas de medición y control automático, como son los actuadores y sensores. Cabe mencionar que los objetivos del protocolo del proyecto se organizan de la siguiente forma: diseño, simulación, e implementación para diversas aplicaciones en el campo industrial, habitacional, aérea, médica, etc., usando nuevas tendencias en MEM, que existen en el mercado mundial. Para ello, a continuación, se discuten los diferentes conocimientos teóricos y experimentales $[4,5]$.

Flujo de capa límite. A continuación, se presenta en la Fig. 1 el flujo de fluidos y sus diferentes capas, donde se puede apreciar que una capa límite consta de tres subcapas importantes: una subcapa viscosa o laminar, una subcapa de transición, y una subcapa turbulenta.

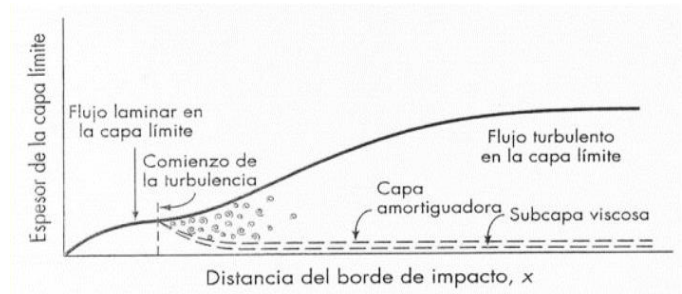

Fig. 1. Espesor de la capa límite en función de la distancia del borde de impacto, las diferentes subcapas. 
Debido a cuerpos que están inmersos en un fluido, las capas límites toman diferentes formas, tal como puede observarse en la Fig. 2.

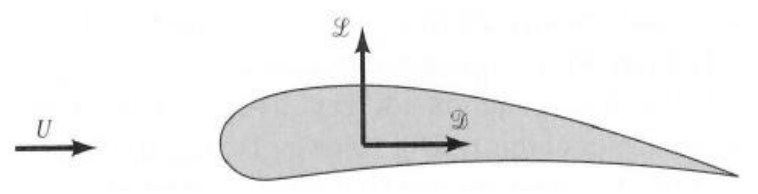

Fig. 2. Resultante de fuerzas horizontal y vertical sobre un perfil alar de un cuerpo dentro de un fluido.

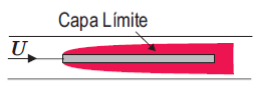

(a) Placa plana delgada

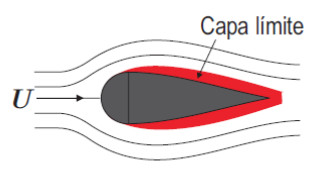

(b) Cuerpo aerodinámico.

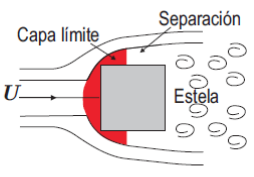

(c) Cuerpo obstructor.

Fig. 3. Capas límites de cuerpos (color rojo) inmersos en fluido. a) Placa plana delgada, b) Cuerpo aerodinámico, c) Cuerpo obstructor.

La magnitud de la capa límite dependerá de la forma que tome el flujo alrededor del cuerpo. La Fig. 3 muestra tres tipos de cuerpos dentro de un fluido, y sus respectivas capas límite están pintadas de rojo.

El cálculo de la capa límite tiene que ver con dos parámetros importantes: el coeficiente de fricción (viscosidad), y su espesor. Estos parámetros difieren fuertemente dependiendo de las capas antes mencionadas [6].

Número de Reynolds. En 1851 George Gabriel Stokes introdujo el concepto de número de Reynolds y fue nombrado por Osborne Reynolds en 1883. El número de Reynolds (véase ecuación 1) es un número adimensional utilizado en mecánica de fluidos, diseño de reactores y fenómenos de transporte para caracterizar el movimiento de un fluido. Su valor indica si el flujo sigue un modelo laminar o turbulento. El número se puede definir como las relaciones entre fuerzas inerciales y las fuerzas viscosas presentes en un fluido. Se puede decir que relaciona la densidad $(\delta)$ y la velocidad (Vs), dimensiones que aparece en muchos cálculos de la dinámica de fluidos, sobre todo en las ecuaciones de Navier Stokes que gobiernan los movimientos de los fluidos. Por ejemplo, si el número vale menos 2100, el flujo es laminar; si va más allá de los 4000 , es flujo turbulento. Así de sencillo:

$$
R e_{x}=\left(\frac{\rho}{\mu} U\right) X
$$

donde $\rho$ =densidad del fluido, $\mu$ =viscocidad dinámica, $U=$ velocidad de corriente libre, $X=$ distancia al borde del ataque.

Coeficientes de fricción. El coeficiente de fricción en un líquido o un gas se le denomina viscosidad y se mide mediante un orificio por donde sale el fluido. La 
velocidad con que este sale es la propiedad de viscosidad de los fluidos (véase la Fig. 4). Como se puede ver, este depende indirectamente de los números de "Reynolds" [7].

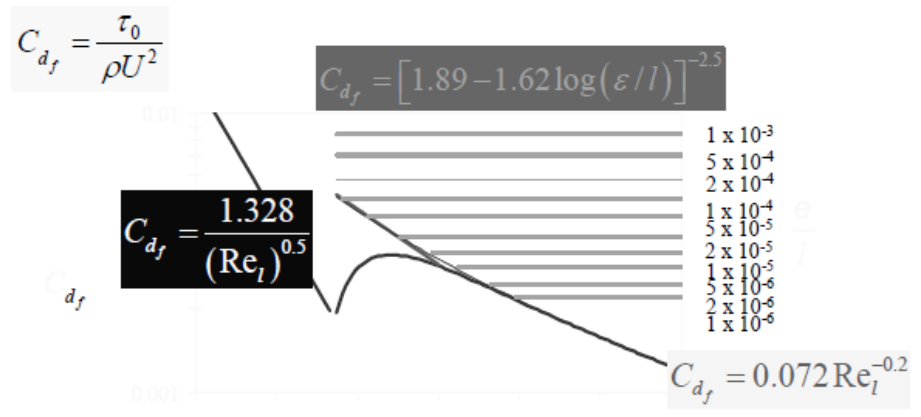

Fig. 4. Coeficientes de fricción en una placa plana usando los números de Reynolds.

Efecto Piezoeléctrico y piezoeléctrico inverso. En 1880, Jacques y Pierre Curie descubrieron que al aplicar presión a un cristal de cuarzo se generaban cargas eléctricas en el cuarzo, a lo que se le llamó "el efecto piezoeléctrico". Más tarde, ellos verificaron que si ahora aplicaban un campo eléctrico al cristal de cuarzo proporcionaba una deformación al material. Este efecto lo llamaron "piezo inverso". De forma tal que se tiene que los materiales piezoeléctricos convierten energía mecánica en eléctrica y viceversa. La palabra piezoeléctrico es una palabra compuesta por piezo y eléctrica, donde "piezo" se deriva de la palabra griega $\pi \iota \zeta \omega \omega$, que significa estrechar, apretar u oprimir.

Las propiedades de los materiales piezoeléctricos son de dos orígenes: naturales y no naturales. Dentro de los naturales está el cuarzo, la turmalina, la sal de Rochelle, etc. A partir de los materiales naturales se han desarrollado los no naturales, mediante una combinación de materiales cerámicos ferro eléctrico policristalino, como el $\mathrm{BaTiO}_{3}, \mathrm{y}$ el Zirconato Titanato de Plomo (PZT). Este último material es muy empleado en aplicaciones como actuadores o sensores. Generalmente los campos eléctricos para efectuar un movimiento mecánico en un material PZT cerámico andan arriba de los $2000 \mathrm{~V} / \mathrm{mm}$.

\section{Métodos}

El diseño y la simulación de control inteligente se llevó a cabo tomando en consideración las leyes de la física de los fluidos y expresados con la teoría de control inteligente, basados la teoría de los conjuntos y la lógica difusa e implementados en bloques usando Simulink-Matlab ${ }^{\circledR}$ para su experimentación mediante gráficos en dos dimensiones. Los cálculos de los parámetros del prototipo de control inteligente se lograron en lazo cerrado (control retroalimentado), cuya variable a controlar fue el flujo de líquido de baja viscosidad entre un recipiente cisterna a un recipiente de producción. La estructura del controlador inteligente está basada en el modelo de Mamdani, el cual contiene tres submodelos importantes: fuzzificador, sistema de inferencia difusa y el defuzzificador. El diseño de los submodelos de Mamdani son como sigue: el submodelo 
de fuzzificador se diseñó con tres funciones de membresía triangulares, el submodelo es parametrizado con inferencia de Mamdani que lleva tres reglas con los conectores lógicos Si, Entonces; el defuzzicador fue hecho también con tres funciones triangulares $[9,10]$.

En la etapa del diseño solamente se requieren los rangos de operación del proceso de microbombas MEM, así como el rango de medición de actuador piezoeléctrico MEM para obtener la simulación del controlador inteligente. Las premisas consideradas a priori son que se considerará un flujo laminar y de baja viscosidad para efectos de diseño y simulación.

El prototipo de implementación consiste en dos modelos: el modelo de Proceso de Fluidos de baja viscosidad, el modelo de Control Inteligente embebido en el modelo electrónico digital, en donde el modelo de Control Inteligente cuenta con Sensor/Actuador basado en tecnología electrónica MEM y va acoplado con "drivers" electrónicos de voltaje medible, lo que permite el monitoreo basado con tecnología Raspberry Pi 3 y pantalla "touch screen" [10].

\subsection{Método de sistema de control inteligente (SCI). Prototipo de simulación gráfica}

La construcción de este sistema se detalla a continuación, mediante las siguientes etapas:

1. Medición de señales: Se utilizaron distintos tipos de dispositivos electrónicos, entre los cuales están: excitador de voltaje tipo Boots, bomba piezoeléctrica MEM, sensor piezo eléctrico MEM y rasberry Pi 3.

2. Adquisición de datos y procesamiento: Las siguientes fases corresponden a la conversión analógico-digital y al procesamiento de la información. En este caso se empleó un microcontrolador Arduino nano, que incluye el control inteligente debido a su capacidad de procesamiento y amplio conjunto de interfaces de comunicación, como son puertos paralelos, números de convertidores analógico-digitales e interfaces de transmisión síncrona o asíncrona.

3. Interfaz: El desplegado de la información se realiza mediante un LCD de 2 líneas por 16 columnas, en donde los valores son mostrados.

\subsection{Modelo de análisis}

El método empleado para la implementación del diseño de Sistema de Control Inteligente fue el de Mamdani, que consiste en tres principales módulos son: Fuzzificador, Base del Conocimiento, y Defuzzificador.

La variable de entrada presenta valores y rangos diferentes, por lo que es necesario transformarlos a un espacio en donde todas tengan un mismo rango de medición. Esto se realiza mediante la aplicación de una función de pertenencia $(\mu)$, la cual estandariza a cada una de las mediciones de cada parámetro en valores entre 0 y 1 . No existe alguna regla que defina cómo construir una función de pertenencia; sin embargo, una función 
lineal simplifica el proceso. Derivado de esto, se diseñaron funciones triangulares, mismas que se pueden expresar mediante la ecuación 2.

$$
\mu(x, a, b, c, d)=\max \left\{0, \min \left[\frac{x-a}{b-a}, 1, \frac{d-x}{d-c}\right]\right\},
$$

donde x representa la variable de entrada; a, b, c y d son los parámetros que definen a la función de entrada, en caso de una función triangular $b=c$.

En el método de fuzzificación se plantea con los mínimos recursos, tres conjuntos para la variable de entrada de Error de Flujo. Mientras que para la Base de Reglas fueron solamente tres reglas Si-Entonces. Finalmente, el defuzzificador solamente se diseña con tres funciones de membresía (FEM). Como se puede observar en la Fig.5.

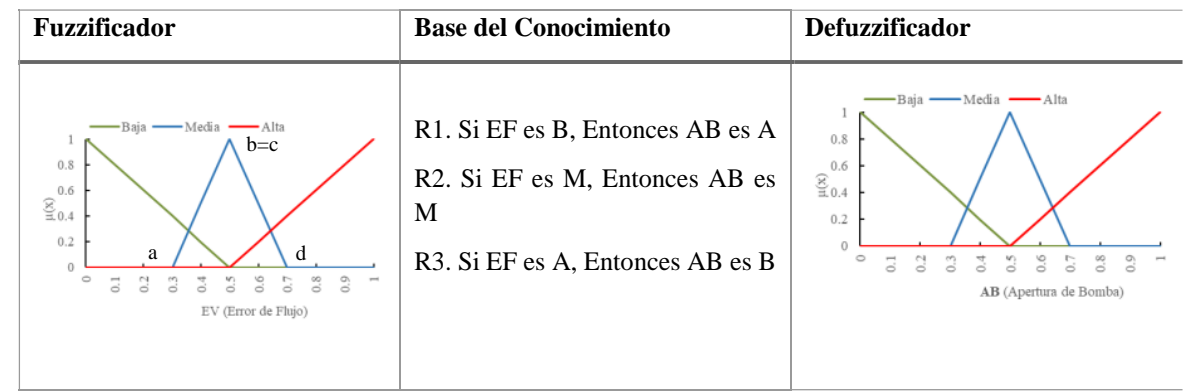

Fig. 5. Método de Mamdani empleado, funciones de Membresía de Entrada/Salida (Error de flujo/Apertura t de Bomba).

\subsection{Prototipo de simulación grafica a través del método de SCI}

En la Fig.6. se presenta la descripción breve de la simulación de las diferentes técnicas de control empleadas para llevar a cabo la comparación de respuestas que se esperan, para determinar la estabilidad de cada uno de ellos y observar de una manera visual las trayectorias [12].

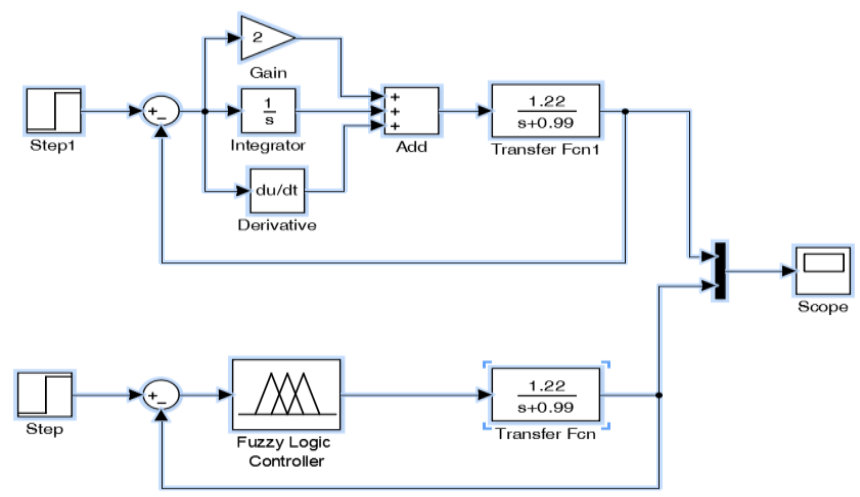

Fig. 6. Diagrama a Bloques de Controlador Clásico PID (a), y controlador inteligente Lazo (b) usando el método de Mamdani (b). 


\subsection{Método de control inteligente. Prototipo de hardware}

Los parámetros encontrados con el método de control inteligente, vía la simulación del apartado anterior, determina el Prototipo de hardware siguiente [13], véase la Fig. 7.

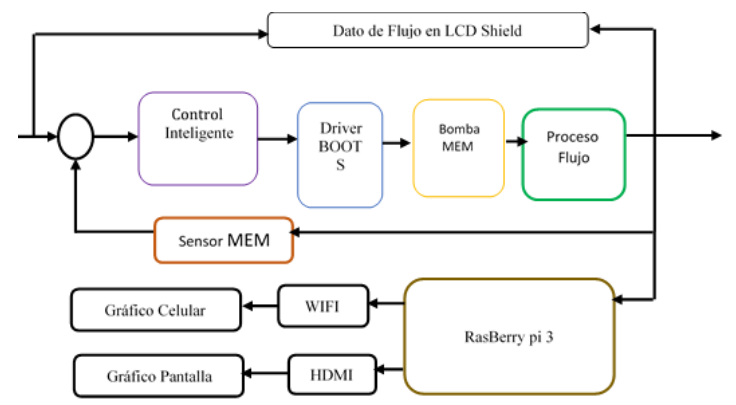

Fig. 7. Diagrama a Bloques de Método de SCI de Flujo de baja Viscosidad.

\section{Resultados}

En el método diseñado empleado para determinar la función de transferencia del proceso de flujo de baja viscosidad, las respuestas de la bomba piezoeléctrica MEM se presenta en la Fig. 8, y la respuesta del sensor de flujo tipo piezoeléctrico MEM está caracterizado por la curva de la Fig. 9.

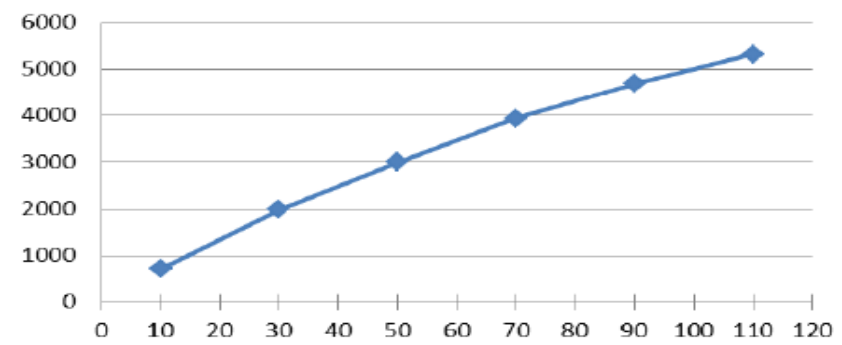

Fig. 8. Gráfico de la Salida de La Bomba MEM (micro Lt/min Vs Frecuencia ciclos/min).

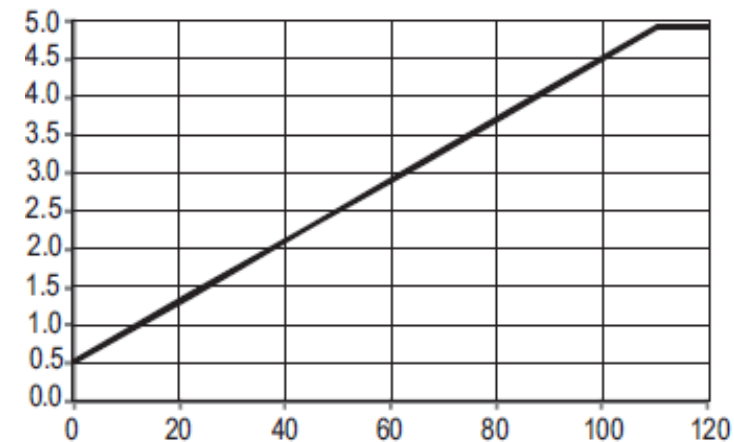

Fig. 9. Gráfico del Sensor de Flujo x = razón de Flujo de Masa, y = voltaje de Salida. 
Ahora seleccionando un punto de operación a $\mathrm{f}=100 \mathrm{~Hz}$ se tiene valores de $5 \mathrm{ml} / \mathrm{min}$ de flujo, correspondiendo a un voltaje de 4.5 voltios. Con ello, el flujo nominal ocupa un tiempo de $0.01 \mathrm{~s}$. Con este valor constante de tiempo del proceso de flujo del fluido a baja viscosidad se tiene una función de transferencia como la que se ve en la Fig. 9. Usando Simulink- Matlab® se diseñó primero para un sistema de control clásico. El control clásico (PID) arroja los rangos de operación de entrada/salida (E/S) del controlador (a). Mismos que servirán para determinar los rangos del Sistema de control Inteligente (b) [11].

\subsection{Resultado de prototipo de simulación usando los métodos de control clásico e inteligente}

La Fig. 10 muestra los resultados del prototipo de Simulación de los Métodos del SCI aplicado a Proceso de Flujo de fluido de baja viscosidad, corriendo a una consigna de $1 \mathrm{ml} /$ minuto. En la figura se puede apreciar la convergencia o estabilidad de ambos métodos de Control de lazo cerrado aproximadamente a $20 \mathrm{~s}$.

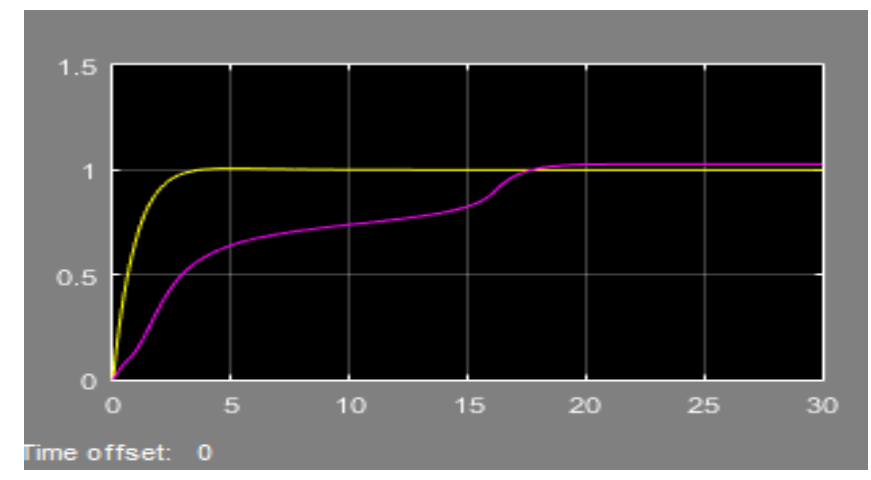

Fig. 10. Respuestas a los sistemas de control Clásico (A)/Inteligente (M). Respuestas a los SCC (amarillo) y SCI(Magenta). Set point Normalizado ( $2 \mathrm{~m} / \mathrm{min})$.

El resultado del Prototipo de hardware usando el método de Control Inteligente, se muestra en la Fig. 11.

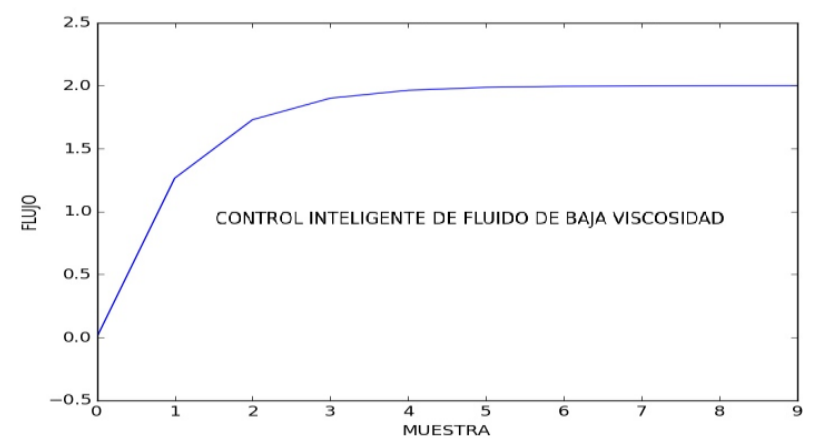

Fig. 11. Respuesta del Método de SCI Aplicado a $2 \mathrm{ml} / \mathrm{min}$ en Pantalla gráfica Rasberry. 


\section{Conclusiones}

El presente trabajo desarrolló una herramienta especializada en la dosificación de líquidos. Su principal ventaja es el control de flujos de baja viscosidad. En comparación con los sistemas existentes en el mercado, este es más accesible ya que su producción involucra un menor costo.

En este trabajo presentamos un nuevo modelo exitoso para controlar la apertura de la bomba MEM para la dosificación de fluidos mediante control inteligente. Las reglas del sistema de control se basaron en el error de flujo, para garantizar la apertura óptima y correcta de las micro bombas MEM. Se definieron funciones de membresía triangulares para normalizar las evaluaciones particulares en un rango de [0 - 1]. De acuerdo con los resultados, el índice propuesto en este artículo demuestra una mejor eficiencia y precisión en comparación con el otro método típico PID. La eficacia y la precisión son muy importantes en la administración de fluidos.

Como trabajo futuro, es importante estudiar las características de otros líquidos para introducir otros tipos de parámetros que deben considerarse. Este trabajo tiene un impacto académico, social y económico, puesto que permite ver la integración de diferentes materias y conocimientos científicos con un fin común y con tecnología de punta a la altura de los países industrializados, y reduce los costos de instalación, mantenimiento, operación y de bajo consumo por usar tecnologías tipo MEM.

Agradecimientos. Los autores agradecen al Centro de Investigación en Computación del Instituto Politécnico Nacional (IPN) y al Consejo Nacional de Ciencia y Tecnología (CONACyT), por su apoyo en el desarrollo de este trabajo, a través de los proyectos SIP20180762 y SIP20181910.

\section{Referencias}

1. Prieto, P.: La innovación, clave de los sistemas de mando y control de Defensa. Homeland Security and Defense (2012)

2. Clyde Warsop: AEROMENS II. Advanced Flow Control Using MEMS Results and Lessons BAE SYSTEM. Learned (2006)

3. Jiang Chengyu, Deng Jinjun, Ma Bringhe, Yuan Weizheng. Advanced flow measurement and active flow control of aircraft with MEMs (2012)

4. Orozco-Lozano, W. A.: Diseño y simulación de las fuerzas de arrastre y sustentación en los autos. Universidad Autónoma del Caribe. Vol. 4. Jul-Dic, pp. 26-33 (2006)

5. Mercado, J.R., Guido, P., Sánchez-Sesma, J., Íñiguez, M.: Fórmulas para el coeficiente de arrastre y la ecuación Navier-Stokes fraccional. Tecnología y Ciencias del Agua. Vol. V, núm. 2, pp. 149-160 (2014)

6. Gherardelli, C.: Mecánica de Fluidos. Cap. 10 (2014)

7. Manuel, F., Mejía de Alba, García-Fernández, L. E., Gutiérrez-Almonacid, M. A.: Metodología de obtención de los coeficientes de sustentación y arrastre para un rango amplio de números de Reynolds y ángulos de ataque para aplicaciones en turbinas eólicas. Universidad de América (2011)

8. Cupich-Rodríguez, M., Elizondo Garza, F. J.: Actuadores piezoeléctricos. 
Midory-Esmeralda Vigueras-Velázquez, Romeo Urbieta-Parrazales, Karen-Alicia Aguilar-Cruz, et al.

9. León-Sandoval, J. J.: Componentes de Sistemas de Control de Flujo y Temperatura para aplicaciones en la Industria de la Fundición. Monterrey N.L. Universidad Autónoma de Nuevo León. Tesis Maestría (1989)

10. García, R. J., Pinto, A. D., Rengel, J. E., Torres, J. M., González, J. A., Pérez, N. A.: Diseño de una estrategia de control difuso aplicada al proceso de ultracongelación de alimentos.

11. Escaler, X., Baliu-Henne, A.: Simulación de la respuesta fluido- dinámica del sistema de refrigeración del sincrotrón ALBA. Detección de aire en tuberías (2016)

12. González-Fontanet, J. G., Haber Guerra, R. E., Matía, F., Novo, M.: Diseño de sistemas de control en cascada clásico y borroso para el seguimiento de trayectorias. Jornada de Automática 2017. Cuba (2017)

13. Servo Flow Corporation: Microcomponents. Application Notes. 